\title{
SOME PROPERTIES OF LAPLACE TRANSFORMS OF MEASURES
}

\author{
BY \\ O. S. ROTHAUS
}

Let $\mu$ be a Borel measure on $\boldsymbol{R}^{n}$, and $\langle.,$.$\rangle the usual scalar product thereon.$ In the first part of this paper we will describe some properties of the Laplace transform

$$
L_{\mu}(x)=\int_{R^{n}} \exp \langle x, t\rangle d \mu_{t}
$$

which arise incidentally in the study of holomorphic functions in tube domains. Some of these properties are not new, or are known in special cases, and we refer the reader to [1], [2] for background. In the second section we make some applications; one of these gives Bergman Kernel for arbitrary tube domains; thus generalizing results of [5], [6].

I. From Hölder's inequality, it is apparent that the set of $x$ for which $L_{\mu}(x)$ is finite is convex. We denote the interior of this set by $F_{\mu}$, and we are principally interested in cases where $F_{\mu}$ is nonempty. The following lemma, whose proof can be found in [1, p. 129], is useful in such cases.

Lemma. Let $p \in F_{\mu}$. There is an $\varepsilon>0$ such that

$$
\int_{R^{n}} \exp \langle p, t\rangle \exp \left(\varepsilon \sum_{i=1}^{n}\left|t^{i}\right|\right) d \mu_{t}<\infty
$$

This result will be used without specific mention to justify the assorted analytic manipulations of the integral for $L_{\mu}(x)$ which we subsequently perform.

Hölder's inequality may be interpreted as saying that $\log L_{\mu}(x)$ is a convex function of $x \in F_{\mu}$; and this implies that the $n \times n$ symmetric matrix

$$
H_{\mu}(x)=\left[\partial^{2} \log L_{\mu}(x) / \partial x^{i} \partial x^{j}\right]
$$

is positive semidefinite. If the convex hull of the support of $\mu$ has a nontrivial interior, then it is easy to see that we only have trivial cases of equality in Hölder's inequality, which suggests that $H_{\mu}(x)$ is positive definite. Now let $S_{\mu}$ be the interior of the convex hull of the support of $\mu$, and define $\mu$ to be satisfactory if both $F_{\mu}$ and $S_{\mu}$ are nonempty. Then we have

Lemma. If $\mu$ is satisfactory, $H_{\mu}(x)$ is positive definite for $x \in F_{\mu}$.

Received by the editors December 19, 1966. 
As a straightforward calculation shows

$$
\begin{aligned}
L_{\mu}^{2}(x) \sum_{i, j} \frac{\partial^{2} \log L_{\mu}(x)}{\partial x^{i} \partial x^{j}} r^{i} r^{j} & =L_{\mu}^{2}(x) \frac{d^{2}}{d \lambda^{2}} \log L_{\mu}(x+\lambda r)(\lambda=0) \\
& =\frac{1}{2} \int\langle r, t-s\rangle^{2} \exp \langle x, s+t\rangle d \mu_{s} d \mu_{t}
\end{aligned}
$$

for any $r \in \boldsymbol{R}^{n}$, which immediately proves the lemma.

We define a mapping $h_{\mu}: F_{\mu} \rightarrow R^{n}$ by setting $h_{\mu}(x)=\operatorname{grad} \log L_{\mu}(x)$. Note that the matrix $H_{\mu}$ gives the Jacobian of the mapping $h_{\mu}$.

LEMMA. If $\mu$ is satisfactory, $h_{\mu}$ is an injection.

Let $r \in \boldsymbol{R}^{n}, r \neq 0, \lambda \in R$. As the last lemma shows,

$$
\left\langle h_{\mu}(x+\lambda r), r\right\rangle=(d / d \lambda) \log L_{\mu}(x+\lambda r)
$$

is a strictly increasing function of $\lambda$, where defined. Put $r=y-x$. Then $x+\lambda(y-x) \in F_{\mu}$ for all $0 \leqq \lambda \leqq 1$. Hence

$$
\begin{aligned}
\left\langle h_{\mu}(y), y-x\right\rangle & =\left\langle h_{\mu}(x+(y-x)), y-x\right\rangle \\
& >\left\langle h_{\mu}(x), y-x\right\rangle .
\end{aligned}
$$

Thus if $y \neq x$, then $h_{\mu}(y) \neq h_{\mu}(x)$.

By virtue of the last result, the range of $h_{\mu}$ becomes of some interest. The determination of the range depends on the boundary behavior of $L_{\mu}(x)$. First we have

Lemma. Let $p$ be a point of $S_{\mu}$. Then $L_{\mu}(x) \exp \langle-x, p\rangle \rightarrow \infty$ as $x \rightarrow \infty$.

Let $B$ be an $n$-simplex contained in $S_{\mu}$ and containing $p$ in its interior. The vertices of $B$ are each convex combinations of finitely many points of support of the measure $\mu$, and $p$ is a convex combination with positive coefficients of the vertices of $B$. So we may write $p=\sum_{i=1}^{k} \lambda_{i} t_{i}, \lambda_{i}>0, t_{i}$ being a point of support for $\mu$, and the convex hull of the $t_{i}$ has a nonempty interior. Let $\varepsilon>0$, and consider the set $T_{\varepsilon}$ of $x \in \boldsymbol{R}^{n}$ for which $\left\langle x, t_{i}-p\right\rangle \leqq \varepsilon|x|, i=1,2, \ldots, k$. We claim $T_{\varepsilon}$ consists only of the origin for sufficiently small $\varepsilon$. Otherwise we can find a point $s$ on the unit sphere for which $\left\langle s, t_{i}-p\right\rangle \leqq 0$ for all $i$. Since $p=\sum \lambda_{i} t_{i}, \lambda_{i}>0$, we conclude $\left\langle s, t_{i}-p\right\rangle=0$, which means of course that $s=0$. This contradiction proves the claim.

Now let $\varepsilon$ be such that $T_{\varepsilon}$ consists only of the origin. Integrating $\exp \langle x, t\rangle d \mu_{t}$ over a disjoint union of spheres, each of radius at most $\varepsilon / 2$, centered at points $t_{1}, t_{2}, \ldots, t_{k}$, we obtain, for suitable positive constant $c$,

or

$$
L_{\mu}(x) \geqq c \exp (-\varepsilon|x| / 2) \sum_{i} \exp \left\langle x, t_{i}\right\rangle
$$

$$
L_{\mu}(x) \exp \langle-x, p\rangle \geqq c \exp (-\varepsilon|x| / 2) \sum_{i} \exp \left\langle x, t_{i}-p\right\rangle
$$


For some $i$ we must have $\exp \left\langle x, t_{i}-p\right\rangle \geqq \varepsilon|x|$. Thus

$$
L_{\mu}(x) \exp \langle-x, p\rangle \geqq c \exp (\varepsilon|x| / 2)
$$

and the lemma is now clear.

Subsequently we will have need of the estimate of the last lemma, rather than the lemma itself.

The complete description of the range of $h_{\mu}$ will be possible only in case of some additional information on the boundary behavior of $L_{\mu}(x)$.

Definition. We say $\mu$ is pioneering if $\mu$ is satisfactory and $L_{\mu}(x)$ approaches infinity as $x$ approaches the finite boundary of $F_{\mu}$.

Note that a satisfactory measure of bounded support is automatically pioneering.

THEOREM. If $\mu$ is pioneering, then $h_{\mu}$ gives a bijection of $F_{\mu}$ with $S_{\mu}$.

We have

$$
\operatorname{grad} \log L_{\mu}(x)=\frac{1}{L_{\mu}(x)} \int t \exp \langle x, t\rangle d \mu_{t}
$$

The expression on the right is a convex combination of all the support points of $\mu$; hence it belongs to $S_{\mu}$. Now we know that $h_{\mu}$ is an injection of $F_{\mu}$ to $S_{\mu}$. To see that $h_{\mu}$ is onto, let $p \in S_{\mu}$, and consider the function on $F_{\mu}$ given by $L_{\mu}(x) \exp \langle-x, p\rangle$. This function approaches infinity as $x$ approaches infinity, or as $x$ approaches the boundary of $F_{\mu}$. Hence the function has a relative minimum in $F_{\mu}$. At such a minimum point $x$ we must have grad $\log L_{\mu}(x)=p$, so the mapping is onto.

The next results give some useful classes of pioneering measures.

THEOREM. Let $C$ be a closed convex set with interior in $\boldsymbol{R}^{n}$, not containing an entire straight line, and $C(t)$ the characteristic function of $C$. The measure $\mu$ for which $d \mu=C(t) d t$ is pioneering.

The proof will in fact describe $F_{\mu}$ precisely. Let $D$ be the cone of $C$; i.e., $D$ is the largest convex cone having the property that for $p \in C$ and any $d \in D, p+d \in C$. Let $D^{*}$ be the dual of $D$; i.e., $D^{*}=\left\{x \in \boldsymbol{R}^{n} \mid\langle x, t\rangle \geqq 0\right.$ for all $\left.t \in D\right\}$. C does not have an entire straight line, nor does $D$; hence $D^{*}$ has a nonempty interior. We will now prove that $F_{\mu}$ is precisely the interior of $-D^{*}$.

Let $h \in$ Int $D^{*}$; thus $\langle h, d\rangle>0$ for any $d \in D, d \neq 0$. Suppose that $\left\langle h, c_{v} /\left|c_{v}\right|\right\rangle \rightarrow 0$ for a sequence $c_{v}$ of points of $C$ approaching $\infty$. The limit points $l$ of the sequence $c_{v} /\left|c_{v}\right|$ can only be nonzero points of $D$. But $\langle h, l\rangle=0$ is a contradiction. We conclude that $\langle h, c\rangle \geqq \rho|c|$ for all $c \in C$ with $|c| \geqq R$, where $\rho$ and $R$ are appropriate positive constants. This last trivially implies the finiteness of $L_{\mu}(-h)$.

On the other hand if $h \in \mathrm{Bd} D^{*}$, then there is a $d \in D, d \neq 0$, so that $\langle h, d\rangle=0$. Let $p$ be an interior point of $C$. We can find linearly independent points $d=v_{1}, v_{2}$, $\ldots, v_{n}$ and a positive number $\varepsilon$ so that the members of the set $T$ of points of the form $p+\lambda_{1} v_{1}+\lambda_{2} v_{2}+\cdots+\lambda_{n} v_{n}$ with $\lambda_{1} \geqq 0$, and $\left|\lambda_{2}\right|,\left|\lambda_{3}\right|, \ldots,\left|\lambda_{n}\right| \leqq \varepsilon$, all belong to $C$. Hence

$$
L_{\mu}(x) \geqq \int_{T} \exp \langle x, t\rangle d t=\alpha \frac{(-1)}{\left\langle x, v_{1}\right\rangle} \frac{\sinh \varepsilon\left\langle x, v_{2}\right\rangle}{\left\langle x, v_{2}\right\rangle} \ldots \frac{\sinh \varepsilon\left\langle x, v_{n}\right\rangle}{\left\langle x, v_{n}\right\rangle}
$$


where $\alpha$ is a positive constant independent of $x$. Thus as $x$ approaches $-h$ through points of - Int $D^{*}, L_{\mu}(x) \rightarrow \infty$ as asserted.

THEOREM. Let $\mu$ be a satisfactory measure. Put $d \eta=d x / L_{\mu}(x)$. Then $\eta$ is a pioneering measure and $S_{\eta}=F_{\mu}, F_{\eta}=S_{\mu}$.

( $L_{\mu}(x)$ is permitted to take the value of $\infty$, and $1 / \infty$ is to be interpreted as 0 .) By definition, $S_{\eta}=F_{\mu}$.

Let $p \in S_{\mu}$. An estimate made earlier gives $L_{\mu}(x) \geqq c \exp (\varepsilon|x| / 2) \exp \langle x, p\rangle$, which shows that $L_{\eta}(p)$ is finite. Thus $S_{\mu} \subset F_{n}$.

Now let $b \in \operatorname{Bd} S_{\mu}$. Pick a supporting hyperplane to $S_{\mu}$ at $b$; i.e., a point $y$ and number $h$ such that $\langle y, s\rangle \leqq h$ for $s \in S_{\mu}$, and $\langle y, b\rangle=h$. Pick a subspace $V$ of $\boldsymbol{R}^{n}$ complementary to that spanned by $y$, in such a fashion that $V \cap F_{\mu}$ is nonempty. Then

$$
\begin{aligned}
L_{\mu}(\lambda y+v) & =\int \exp \langle\lambda y+v, t\rangle d \mu_{t} \\
& \leqq L_{\mu}(v) \exp (\lambda h) \quad \text { for } \lambda \geqq 0 .
\end{aligned}
$$

Thus

$$
\begin{aligned}
L_{n}(t) & =\int \exp \langle x, t\rangle \frac{1}{L_{\mu}(x)} d x \\
& =\alpha \int \exp \langle\lambda y+v, t\rangle \frac{1}{L_{\mu}(\lambda y+v)} d \lambda d v \\
& \geqq \alpha \int_{0}^{\infty} \exp (\lambda\langle y, t\rangle-\lambda h) d \lambda \cdot \int_{V} \exp \langle v, t\rangle \frac{1}{L_{\mu}(v)} d v \\
& =\alpha \int_{V} \exp \langle v, t\rangle \frac{1}{L_{\mu}(v)} d v \cdot \frac{1}{h-\langle y, t\rangle}
\end{aligned}
$$

where $\alpha$ is the Jacobian arising from the linear transformation of coordinates. Recalling that $L_{\mu}(v)$ is not identically infinity, it is clear from the above that $L_{\eta}(t) \rightarrow \infty$ as $t \rightarrow b$.

II. There is a direct though strange connection of our first theorem with the Brunn-Minkowski Theorem [3]. Namely, let $\mu$ and $\eta$ both be measures in $\boldsymbol{R}^{n}$, each of bounded support, and $S_{\mu}$ and $S_{\eta}$ be nonempty. Using the Titchmarsh convolution theorem, it is easy to see that

$$
S_{\mu \otimes \eta}=S_{\mu}+S_{\eta}
$$

where $\mu \otimes \eta$ is convolution, and $S_{\mu}+S_{\eta}$ is set of all sums of points of $S_{\mu}$ with points of $S_{\eta}$. From our first theorem, it follows that

$$
V\left(S_{\mu}\right)=\text { volume of } S_{\mu}=\int_{R^{n}}\left|H_{\mu}(x)\right| d x .
$$


Also we know $L_{\mu \otimes_{n}}(x)=L_{\mu}(x) \cdot L_{n}(x)$. Now for convex sets $A$ and $B$, the BrunnMinkowski Theorem asserts $V(A+B)^{1 / n} \geqq V(A)^{1 / n}+V(B)^{1 / n}$. Hence we must have

$$
\left[\int_{R^{n}} \mid\left[H_{\mu}(x)+H_{n}(x) \mid d x\right]^{1 / n} \geqq\left[\int\left|H_{\mu}(x)\right| d x\right]^{1 / n}+\left[\int\left|H_{n}(x)\right| d x\right]^{1 / n} .\right.
$$

It does not seem easy to give a direct proof of the last inequality!

As another application of our results, we can easily give the Bergman Kernel Function for tube domains, thus extending [5], [6]. For this purpose let $D$ be an arbitrary connected domain in $R^{n}$ and $T_{D}$ the tube in $C^{n}$ with $D$ as base. By $L_{2}\left(T_{D}\right)$ we denote the space of functions holomorphic and square integrable on $T_{D}$.

Let $L(x)=\int_{D} \exp \langle x, t\rangle d t$. By a minor extension of the argument used in [6], which already leaned heavily on [1], it is easy to show that corresponding to every $f$ in $L_{2}\left(T_{D}\right)$ there is a unique complex valued function $\varphi$ on $\boldsymbol{R}^{n}$ such that

$$
f(z)=\int_{R^{n}} \exp \langle z, t\rangle \varphi(t) d t
$$

and moreover

$$
\int_{R^{n}}|\varphi(t)|^{2} L(2 t) d t<\infty .
$$

(If $L(2 t)$ is almost everywhere infinite, then $L_{2}\left(T_{D}\right)$ consists only of the zero function, and there is nothing to prove.)

Conversely, corresponding to every $\varphi$ with the stated square integrability property, the integral

$$
f(z)=\int \exp \langle z, t\rangle \varphi(t) d t
$$

equals a square integrable holomorphic function in $L_{2}\left(T_{D}\right)$, as the estimates of $\S I$ readily show.

An easy computation, using Plancherel's theorem, also shows that

$$
\int_{T_{D}}|f(z)|^{2} d x d y=(2 \pi)^{n} \int_{R^{n}}|\varphi(t)|^{2} L(2 t) d t
$$

so the correspondence is, except for scaling, an isometry of Hilbert spaces, generalizing the observation made in [5].

Now put

$$
B(x)=\int_{R^{n}} \exp \langle x, t\rangle \frac{1}{L(2 t)} d t .
$$

From the last theorem of $\S \mathrm{I}$, it follows that $B(x)$ is finite for $x \in D+D$. Let $z$, $w \in T_{D}, z=x+i y$. Then we have just seen that $B(w+\bar{z})$ is defined. And furthermore, with the estimates of $\S \mathrm{I}$ available, we find that $B(w+\bar{z})$ is a square integrable 
function of $y$, as well as a square integrable function of $x$ and $y$. Using Plancherel's theorem:

$$
\begin{aligned}
\int_{T_{D}} B(w+\bar{z}) f(z) d x d y & =(2 \pi)^{n} \int_{R^{n} \times R^{n}} \exp \langle w, t\rangle \exp \langle 2 x, t\rangle \varphi(t) L(2 t) d t d x \\
& =(2 \pi)^{n} \int \exp \langle w, t\rangle \varphi(t) d t=(2 \pi)^{n} f(w) .
\end{aligned}
$$

Thus we have

TheOREM. $B(w+\bar{z}) /(2 \pi)^{n}$ is the Bergman Kernel Function for $L_{2}\left(T_{D}\right)$.

A slight alteration of the arguments used to obtain the last theorem will yield results which overlap but are not completely included in statements of Gindikin [4] on Cauchy-Weil Kernels for tube domains. For this purpose, we must define an appropriate operational class of holomorphic functions.

Let $\mu$ be a satisfactory measure in $\boldsymbol{R}^{n}$. We consider the class $C_{\mu}$ of holomorphic functions $f(z)$ in $T_{S_{\mu}}$ having the following two properties:

(i) $\lambda(x)=\int_{R^{n}}|f(x+i y)|^{2} d y$ exists for $x \in S_{\mu}$, and is uniformly bounded for $x$ in any compactum in $S_{\mu}$.

(ii) $\int \lambda(x) d \mu<\infty$.

A word of explanation about condition (ii) is needed. Condition (i) is known to imply the existence of a function $\varphi$ such that $f(z)=\int \exp \langle z, t\rangle \varphi(t) d t$, valid for $z \in T_{S_{\mu}}$ [1]. So for $x \in S_{\mu}$, Plancherel's theorem gives

$$
\lambda(x)=(2 \pi)^{n} \int \exp \langle 2 x, t\rangle|\varphi(t)|^{2} d t
$$

and this equation permits the extension of $\lambda(x)$ to a function on $\boldsymbol{R}^{n}$, possibly taking the value $+\infty$. It is for the extended function $\lambda(x)$ that condition (ii) is to be understood.

Let $\eta$ be the measure on $\boldsymbol{R}^{n} \times \boldsymbol{R}^{n}$ such that $d \eta=d \mu_{x} \cdot d y$. The function $\exp \langle x, y\rangle \varphi(y)$ is $\eta$ measurable; and condition (ii) above may be restated as

$$
\int|\exp \langle x, y\rangle \varphi(y)|^{2} d \eta<\infty
$$

or

$$
\int|\varphi(y)|^{2} L_{\mu}(2 y) d y<\infty
$$

We want to reinterpret condition (ii) in terms of the function $f(z)$ itself. To this end, let $B_{v}$ be the ball of radius $v$ about the origin in $\boldsymbol{R}^{n}$, and put

$$
f_{v}(z)=\int_{B_{v}} \exp \langle z, t\rangle \varphi(t) d t .
$$

The functions $f_{v}(z)=f_{v}(x+i y)$ are continuous in $\boldsymbol{R}^{n} \times \boldsymbol{R}^{n}$, and square summable 
with respect to the measure $\eta$. Furthermore the sequence $f_{v}(z), \nu=1,2, \ldots$ is Cauchy in the space of square integrable functions with respect to the measure $\eta$. This last assertion follows trivially with use of Plancherel's theorem. Hence there is a limit function, $\eta$ square integrable, which we again denote by $f(z)$, since we may suppose it agrees with $f(z)$ in $T_{S_{\mu}}$. Since the values of $f(z)$ outside the support of $\eta$ are irrelevant, it is natural to say that $f(z)$ subject to condition (ii) has generalized boundary values.

Finally we define

$$
K(x)=\int_{R^{n}} \exp \langle x, t\rangle \frac{1}{L_{\mu}(2 t)} d t .
$$

Let $S$ be the support of the measure $\mu$. Using the estimates of $\S I$, it is easy to see that $K(w+\bar{z})$ is well defined and continuous for $w \in T_{S_{\mu}}$ and $z \in T_{S}$. Even more, $K(w+\bar{z})$ is $\eta$ square summable as function of $z=x+i y$. Hence, repeating the argument used for Bergman Kernel, we conclude

$$
f(w)=\frac{1}{(2 \pi)^{n}} \int_{T_{S}} K(w+\bar{z}) f(z) d \eta .
$$

If the measure $\mu$ is concentrated on the boundary of $S_{\mu}$, then the formula above may be viewed as a version of Cauchy's formula.

\section{REFERENCES}

1. S. Bochner and W. T. Martin, Several complex variables, Princeton Univ. Press, Princeton, N. J., 1948.

2. S. Bochner, Group invariance of Cauchy's formula in several variables, Ann. of Math. (2) 45 (1944), 686-707.

3. T. Bonnesen and W. Fenchel, Theorie der Convexen Körper, Springer, Berlin, 1934.

4. S. G. Gindikin, Analytic functions in tube domains, Dokl. Akad. Nauk SSSR 145 (1962), 1205-1208 = Soviet Math. Dokl. 3 (1962), 1178.

5. A. Korányi, The Bergman kernel function for tubes over convex cones, Pacific J. Math. 12 (1962), 1355-1359.

6. O. S. Rothaus, Domains of positivity, Abh. Math. Sem. Univ. Hamburg 24 (1960), 189-235.

CORNELl UNIVERSITY, ITHACA, NEW YORK 